\title{
Immunophenotypic Analysis of B Lymphocytes in Patients with Common Variable Immunodeficiency: Identification of CD23 as a Useful Marker in the Definition of the Disease
}

\author{
Giuseppe Patuzzo, ${ }^{1}$ Filippo Mazzi, ${ }^{1}$ Antonio Vella, ${ }^{2}$ Riccardo Ortolani, ${ }^{2}$ \\ Alessandro Barbieri, ${ }^{1}$ Elisa Tinazzi, ${ }^{1}$ Giacomo Marchi, ${ }^{1}$ Orazio Codella, ${ }^{1}$ Ruggero Beri, ${ }^{1}$ \\ Antonio Puccetti, ${ }^{3}$ and Claudio Lunardi ${ }^{1}$ \\ ${ }^{1}$ Department of Medicine, Unit of Clinical Immunology and Allergy, University of Verona, 37134 Verona, Italy \\ ${ }^{2}$ Department of Pathology and Diagnosis, Section of Immunology, University of Verona, 37134 Verona, Italy \\ ${ }^{3}$ Institute G. Gaslini and Department of Experimental Medicine, University of Genova, 16132 Genova, Italy
}

Correspondence should be addressed to Claudio Lunardi; claudio.lunardi@univr.it

Received 20 February 2013; Accepted 17 March 2013

Academic Editors: C. M. Artlett, E. K. Kapsogeorgou, and B. E. Palmer

\begin{abstract}
Copyright (C) 2013 Giuseppe Patuzzo et al. This is an open access article distributed under the Creative Commons Attribution License, which permits unrestricted use, distribution, and reproduction in any medium, provided the original work is properly cited.

Common variable immunodeficiency (CVID) is a primary immunodeficiency characterized by the failure of B lymphocytes differentiation leading to deficient immunoglobulins secretion. The identified genetic defects account only for a minority of cases. The importance of B cells immunophenotyping in the classification of CVID is known. This procedure can identify alterations on the cell surface molecules expression that could explain some immunological disorders characteristic of CVID. Moreover, some immunophenotypical aspects can correlate with clinical features of the disease. We used this procedure to analyze a cohort of 23 patients affected by CVID, in order to identify the novel alterations of B cells and to find the possible correlations with clinical features. Circulating B cells were studied by flow cytometry incubating whole blood with specific antibodies for B cell surface molecules including CD27, IgM, IgD, CD21, and CD23. We compared the population of "switched memory" IgD- CD27+ B lymphocytes with the population of "switched memory" IgM- IgD- CD23- CD27+ B cells. These last B cells were reduced in patients compared to healthy controls; moreover, IgM- IgD- CD23-CD27+ B cells were lower than IgD- CD27+ B cells in patients with CVID. The reduction of this subset of B lymphocytes correlates more tightly than IgD-CD27+ B cells with lymphadenopathy and airways infections. In conclusion, our findings may help in better identifying patients with CVID.
\end{abstract}

\section{Introduction}

Common variable immunodeficiency (CVID) is the most common primary immunodeficiency in clinical practice. CVID represents a large heterogeneous group of syndromes characterized by low serum levels of IgG, IgA, and/or IgM, with decreased antibody production and impaired antibody response to both polysaccharide and protein antigens [13]. As a result of low antibody levels, most CVID patients have recurrent airway infections by capsulated bacteria. Autoimmune diseases, lymphoproliferative, granulomatous or neoplastic disorders, and intestinal dysfunctions may also affect CVID patients.
The majority of the genetic mechanisms leading to CVID are still unclear. Defects in the genes that encode for inducible costimulator (ICOS), transmembrane activator and CAML interactor (TACI), CD19, B-cell activating factor receptor (BAFFR), CD81, CD20, and CD21 have been reported [1, 4-6]. The identification and analysis of these gene defects have led to novel hypothesis on the pathogenesis of CVID. However, gene defects have been identified in less than $10 \%$ of patients, and therefore they account only for a small part of the cases of CVID [7-10].

The typical defect of CVID is the failure of B lymphocytes to differentiate into switched memory B cells and into plasma cells. During the different steps of their maturation, B cells 
circulate between splenic follicles, lymph nodes, and bone marrow, until they undergo apoptosis or are activated by an antigen. This allows naïve $\mathrm{B}$ cells to complete their maturation process to switched memory B cells.

In the spleen, immature $B$ cells begin to express the CD21 and CD23 molecules on their surface. CD21 is the receptor for the C3 fraction of complement and is involved in signal transduction pathways triggered by the $\mathrm{B}$ cell receptor (BCR) with CD19 and CD81. CD23 is the low affinity receptor for IgE and plays an important role in the interaction between $\mathrm{B}$ cells and dendritic cells [11]. Upon activation by an antigen, B cells differentiate either into plasma cells [12] or into memory $\mathrm{B}$ cells. During these steps, surface expression of CD23 decreases, and eventually it disappears. Therefore, CD23 can be considered a marker of $\mathrm{B}$ cell activation [13], and it is absent in "switched memory" B cells [14].

One of the characteristics of normally activated B cells that have undergone somatic hypermutation and are able to produce Immunoglobulins (Igs) in response to antigen recall is the presence of $\mathrm{CD} 27$ on their surface; $\mathrm{CD} 27$ is a type 1 TNF family member, considered a marker of memory B cells. In CVID, alterations of the somatic hypermutation process both in the $\operatorname{Ig}(\mathrm{V})$ regions and in the light chains have been described, indicating alterations in B cell maturation in the germinal center [15].

Since abnormalities in peripheral blood B cell subsets are known to be present in patients with CVID [16-19], guidelines support the importance of B cells immunophenotyping with the aim of better identifying patients with CVID. Indeed particular immunophenotypical aspects can be correlated to clinical features and to the severity and prognosis of the disease.

Several studies have been performed in order to describe the immunophenotypical alterations of B cells in CVID. Warnatz et al. in 2002 proposed the "Classification of Freiburg" [16]; Piqueras et al. in 2003 suggested the "Classification of Paris" [17], and more recently Wehr et al. with the EUROclass trial have tried to unify and improve the already existing classification schemes to identify potential prognostic markers for the disease [18]. All the classifications of CVID [16-18] report a good correlation between particular B cell subsets and the main clinical features of the disease. However, they do not consider all the possible aspects and clinical presentations of CVID.

In this work, we performed an immunophenotypical analysis of B cells in a cohort of 23 patients with CVID, in order to identify possible markers of B cells that may correlate with clinical features of the disease.

\section{Methods}

2.1. Patients and Controls. We studied a cohort of 23 patients ( 7 males and 16 females, mean age of $44,8 \pm 12$ years) affected by CVID, attending the Unit of Autoimmune Diseases, Section of Internal Medicine B, at the University Hospital of Verona, Italy. All patients fulfilled the ESID/PAGID criteria for the diagnosis of CVID: marked decrease in IgG levels (at least 2 SD below the mean for patient's age) and marked decrease in at least one of the isotypes $\operatorname{IgM}$ or $\operatorname{IgA}$, plus (1) onset of immunodeficiency after 2 years of age; (2) poor response to vaccines; and (3) exclusion of other defined causes of hypogammaglobulinemia [20].

The levels of Igs at diagnosis and at the moment of enrolment are reported in Table 1.

Eighteen of 23 patients with frequent infections were treated with regular monthly infusion of Igs $(0.4 \mathrm{~g} / \mathrm{kg})$ for a period of $10,55 \pm 7,28$ years.

When enrolled, all the patients underwent clinical examination, laboratory evaluation, including $B$ cells immunophenotyping and chest X-ray, abdominal ultrasound, and high resolution-computed tomography (HRCT) in some cases.

At enrollment, none of the patients had active infections or was treated with antineoplastic or immunosuppressive drugs. Moreover, none suffered from malignancies. Patients with a past medical history of cancer did not show any sign of disease relapse at least for 5 years.

The clinical features of CVID patients considered in this study are shown in Table 2. In particular, we have considered: the presence of infections; autoimmune diseases such as autoimmune cytopenia, thyroiditis, arthritis; malignancies; splenomegaly, detected by ultrasonography; lymphadenopathy, detected by clinical examination, by ultrasonography or computed tomography (CT); bronchiectasis, identified by HRCT; and the Ig levels at the time of diagnosis and of enrollment in the study and the immunophenotypical study.

Eleven age- and sex- matched healthy donors served as controls.

A written informed consent was obtained from all the participants to the study. The study was approved by local Ethical Committee.

2.2. Flow Cytometry and B Lymphocytes Subpopulations. Blood samples were collected from patients and controls in EDTA tubes. Patients receiving Igs were bled 27 days after the last infusion of Igs.

Two tubes containing $100 \mu \mathrm{L}$ of whole blood were prepared. The samples were diluted with $200 \mu \mathrm{L}$ of saline isotonic buffer and were centrifuged. Plasma was removed by Lyse/Wash Assistant (B Biosciences), and $200 \mu \mathrm{L}$ of each sample were incubated for 20 minutes at $37^{\circ} \mathrm{C}$ either with antibodies anti-IgD FITC, -IgM PE Cy5, -CD23 PE, -CD27 APC, -CD5 PE Cy7, and -CD19 APC Cy7 (BD Biosciences), or with antibodies anti-IgD FITC, -IgM PE Cy5, -CD21 PE, -CD27 APC, -CD5 PE Cy7, and -CD19 APC Cy7 (BD Biosciences). After incubation, red blood cells were lysed by the Lyse/No Wash Procedure, using BD-FACS lysing solution (BD Biosciences) with $\mathrm{BD}$ Lyse/Wash Assistant (BD Biosciences). Both samples were analyzed by a sixcolor FACScanto cytometer (BD Biosciences); 2000 cells per sample were acquired in the region of CD19+ CD5$B$ lymphocytes, which represent the majority of circulating B cells. FACSDiva software (BD Biosciences) was used to analyze data.

2.3. Statistical Analysis. Statistical analysis was performed with SPSS 20.0 statistical package (SPSS Inc. Chicago, IL, 
TABLE 1: Levels of immunoglobulins at diagnosis and at the moment of the immunophenotypic analysis (levels of immunoglobulins are expressed as mean $\pm \mathrm{SD}$ ).

\begin{tabular}{lcccc}
\hline \multirow{2}{*}{ Immunoglobulins } & \multicolumn{2}{c}{$\begin{array}{c}\text { Levels at diagnosis } \\
(\mathrm{g} / \mathrm{L})\end{array}$} & \multicolumn{2}{c}{$\begin{array}{c}\text { Levels at the moment } \\
\text { of enrollment }(\mathrm{g} / \mathrm{L})\end{array}$} \\
\cline { 2 - 5 } & Male & Female & Male & Female \\
\hline IgG & $2.33 \pm 1.32$ & $2 \pm 1.08$ & $5 \pm 3.38$ & $7.7 \pm 2.77$ \\
IgA & $0.07 \pm 0.1$ & $0.14 \pm 0.24$ & $0.05 \pm 0.05$ & $0.19 \pm 0.29$ \\
IgM & $0.32 \pm 0.6$ & $0.83 \pm 0.57$ & $0.51 \pm 0.12$ & $0.21 \pm 0.27$ \\
\hline
\end{tabular}

TABLE 2: Clinical features of the patients with CVID considered in the study.

\begin{tabular}{lc}
\hline Clinical features & $\begin{array}{c}\text { Patients number } \\
\text { (percentage) }\end{array}$ \\
\hline Infections & $23(100 \%)$ \\
Airways infections & $23(100 \%)$ \\
Gastrointestinal infections & $8(34 \%)$ \\
Genitourinary infections & $7(30 \%)$ \\
Conjunctivitis & $2(9 \%)$ \\
Otitis & $4(17 \%)$ \\
Esophageal candidiasis & $3(13 \%)$ \\
Poliomyelitis & $1(4 \%)$ \\
Meningitis & $1(4 \%)$ \\
Bronchiectasis & $6(26 \%)$ \\
Splenomegaly & $7(30 \%)$ \\
Lymphadenopathy & $10(43 \%)$ \\
Haematological malignancy & $1(4 \%)$ \\
Hodgkin's lymphoma & $1(4 \%)$ \\
Autoimmune diseases & $12(52 \%)$ \\
Hypothyroidism/thyroiditis & $5(22 \%)$ \\
Autoimmune thrombocytopenia & $4(17 \%)$ \\
Autoimmune anemia & $1(4 \%)$ \\
Sjögren's syndrome & $3(13 \%)$ \\
Arthritis & $2(9 \%)$ \\
Celiac sprue & $1(4 \%)$ \\
Alopecia & $1(4 \%)$ \\
Sarcoidosis & $1(4 \%)$ \\
Lichen planus & $1(4 \%)$ \\
Beoplasias & $7(30 \%)$ \\
Genital cancer & $2(13 \%)$ \\
Basolon cancer & $2(13 \%)$ \\
Meningioma & $1(6 \%)$ \\
Osteosarcoma & $1(6 \%)$ \\
& $1(6 \%)$ \\
Bust cancer & $1(6 \%)$ \\
\hline
\end{tabular}

USA). Qualitative data with a normal distribution were expressed as mean \pm standard deviation and were analyzed with Student's $t$-test. Data which presented a not-normal distribution were analyzed with not-parametric Mann-Whitney test. A multiple logistic regression analysis that simultaneously considered IgD- CD27+ B cells or IgM- IgDCD23- CD27+ B cells and clinical features was performed to evaluate the independent association between these variables and immunodeficiency. A $P$ value $<0.05$ was considered significant.

\section{Results}

The number of CD19+ CD5- B cells was lower in patients than in controls $(126,02 \pm 108,85$ cells/ $\mu \mathrm{L}$ versus $201,86 \pm$ $75,79$ cells $/ \mu \mathrm{L} ; P<0.05)$.

The B cell population was then evaluated on the basis of the surface expression of CD23 and CD27, and we identified 3 different lymphocytes subsets: CD23+ CD27-; CD23CD27-, and CD23- CD27+ (Figures 1(a)-1(c)).

There was no difference in CD23+ CD27- B cell number between patients and controls $(52,82 \pm 55,23$ cells $/ \mu \mathrm{L}$ versus $82,56 \pm 47,14$ cells $/ \mu \mathrm{L} ; P=0.14$ ) (Figure $1(\mathrm{a})$ ), and there was no correlation between these cells and clinical features of the disease.

Similarly, we did not find a significant difference in

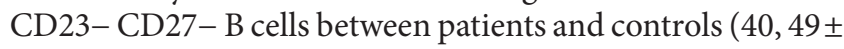
$38,94$ cells $/ \mu \mathrm{L}$ versus $40,68 \pm 24,16$ cells $/ \mu \mathrm{L} ; P=0.9)$ (Figure 1(b)). However, the number of CD23- CD27- B cells was lower in patients with bronchiectasis $(29,65 \pm$ $15,58$ cells/ $\mu \mathrm{L}$ versus $55,37 \pm 47,5$ cells $/ \mu \mathrm{L} ; P=0.04)$ and with malignancy $(17,78 \pm 16,29$ cells/ $\mu \mathrm{L}$ versus $51,85 \pm$ $42,56$ cells $/ \mu \mathrm{L} ; P=0.03)$.

The CD23- CD27+ B cell numbers were significantly decreased in patients compared to controls $(34,36 \pm$ $56,50$ cells $/ \mu \mathrm{L}$ versus $66,39 \pm 34,74$ cells $/ \mu \mathrm{L} ; P<0.05)$ (Figure 1(c)), but there was no correlation between the number of these cells and the presence of bronchiectasis, splenomegaly, malignancy, and autoimmune diseases.

In order to identify further differences among these three B cell populations, we divided them according the IgM and IgD expression. Therefore, we considered the following B cell subsets: IgM+ IgD+ CD23+ CD27- (naïve B cells), IgM+ IgD- CD23- CD27-, IgM- IgD- CD23- CD27-, and IgMIgD-CD23- CD27+ (switched memory B cells).

We did not find differences in the number of $\operatorname{IgM}+\operatorname{IgD}+$ CD23+ CD27- B cells between patients and controls (44, 42 \pm $51,92$ cells $/ \mu \mathrm{L}$ versus $68,30 \pm 43,70$ cells $/ \mu \mathrm{L} ; P=0.19)$ (Figure 2(a)). However, the number of naïve $B$ cells is reduced in patients with splenomegaly $(29,38 \pm 21,12$ cells $/ \mu \mathrm{L}$ versus $56,11 \pm 66,01$ cells $/ \mu \mathrm{L}, P=0.05)$ and lymphadenopathy $(20,68 \pm 20,53$ cells $/ \mu \mathrm{L}$ versus $83,96 \pm 65,77$ cells $/ \mu \mathrm{L}, P<$ $0.05)$, whereas no correlation was found with the presence of autoimmune diseases $(P=0.53)$.

The number of IgM+ IgD- CD23- CD27- B cells is slightly higher in patients than in controls but without statistical significance (Figure 2(b)), whereas IgM- IgD-CD23CD27- B cells are significantly less in CVID patients than in controls $(5,95 \pm 11,23$ cells $/ \mu \mathrm{L}$ versus $13,05 \pm 10,37$ cells $/ \mu \mathrm{L}$; $P<0.05$ ) (Figure 2(c)).

Finally, the number of IgM- IgD-CD23-CD27+ B cells was much lower in patients than in controls $(2,07 \pm$ 


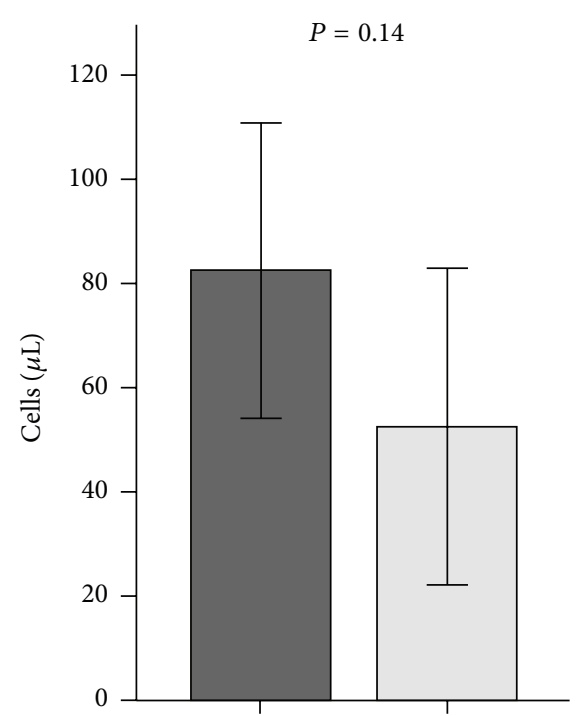

(a)

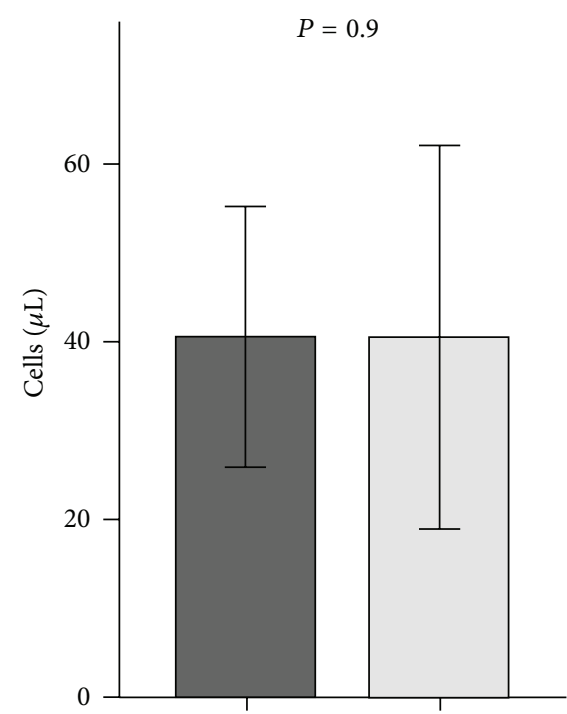

(b)

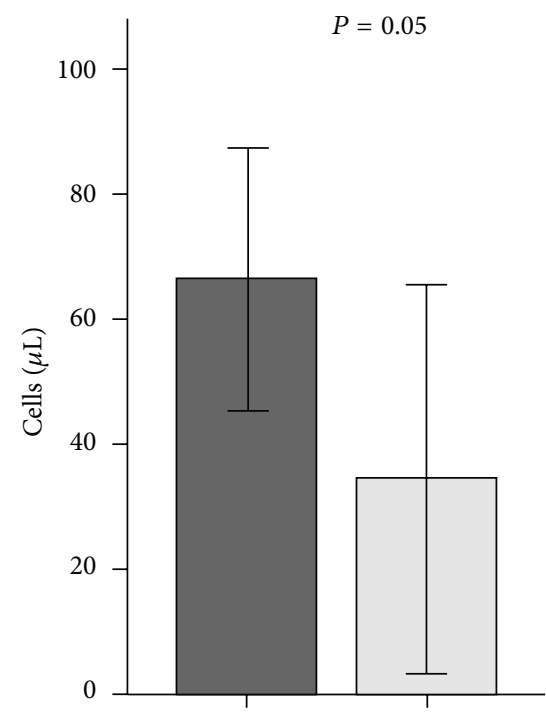

(c)

FIGURE 1: CD23 and CD27 expressions by B lymphocytes in patients with CVID. Number of CD23+ CD27- B lymphocytes (a), of CD23CD27- B lymphocytes (b), and of CD23- CD27+ B lymphocytes (c) in healthy controls (dark grey bar) and in CVID patients (light grey bar). Number of cells are reported on the vertical axis.

$4,8$ cells $/ \mu \mathrm{L}$ versus $28,67 \pm 15,33$ cells $/ \mu \mathrm{L} ; P<0.01)$ (Figure 2(d)), as for IgD- CD27+ B cells used in the Freiburg classification, $(5,96 \pm 8,58$ cells/ $\mu \mathrm{L}$ versus $38,97 \pm$ 19,43 cells $/ \mu \mathrm{L}, P<0.05$ ) (Figure 3 ).

We then compared IgD-CD27+ with IgM- IgD-CD23CD27+ $\mathrm{B}$ cells in CVID patients, and we found that the number of IgD-CD27+ B cells was higher than IgM- IgDCD23- CD27+ B cells $(5,96 \pm 8,58$ cells $/ \mu \mathrm{L}$ versus $2,07 \pm$ $4,80$ cells $/ \mu \mathrm{L} ; P<0.05)$ (Figure 4 ).

The low number of IgD-CD27+ B cells did not correlate with autoimmune diseases $(P=0.69)$, splenomegaly $(P=$ $0.52)$, or lymphadenopathy $(P=0.12)$.
The IgM- IgD- CD23- CD27+ B cells number did not correlate with autoimmune diseases $(P=0.48)$, splenomegaly $(P=0.21)$, and bronchiectasis $(P=0.82)$, whereas it correlated with lymphadenopathy $(0,34 \pm 0,37$ cells/ $\mu \mathrm{L}$ versus $4,95 \pm 7,28$ cells $/ \mu \mathrm{L} ; P=0.05)$ and airways infections $(P=$ $0.04)$ more closely than the number of the IgD-CD27+ B cell subset $(P<0.01$ versus $P=0.03)$.

Finally, we analysed the IgM+ IgD+CD23-CD27+ B cells (also known as marginal zone $\mathrm{B}$ cells) and found that the number of this cell subset is similar in patients and controls $(27,43 \pm 50,45$ cells $/ \mu \mathrm{L}$ and $30,95 \pm 16,09$ cells $/ \mu \mathrm{L})$. The decrease of IgM+ IgD+CD23-CD27+ B cells correlated with 


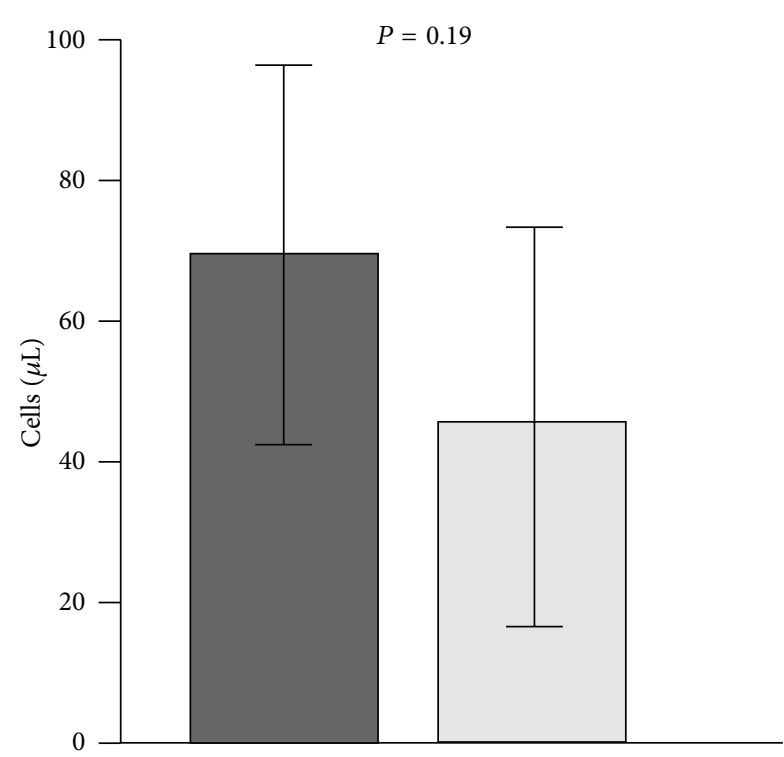

(a)

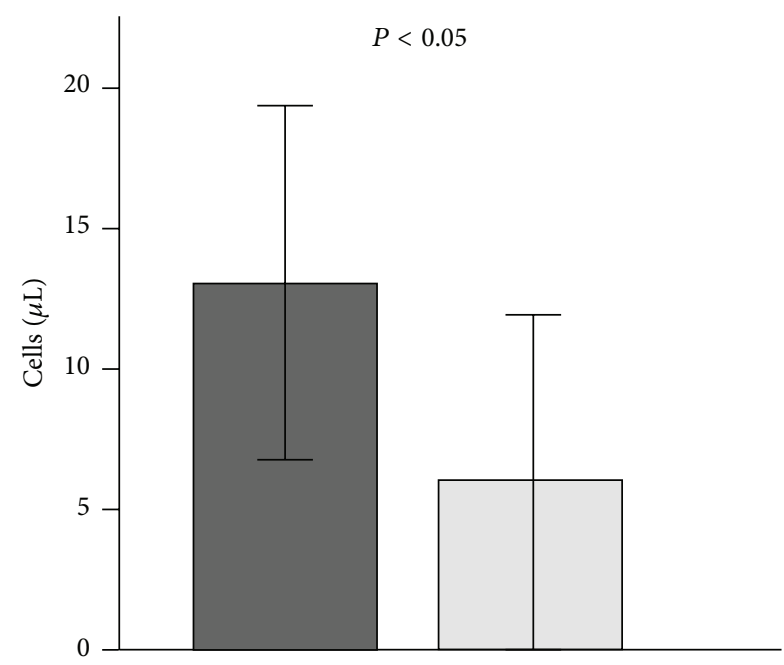

(c)

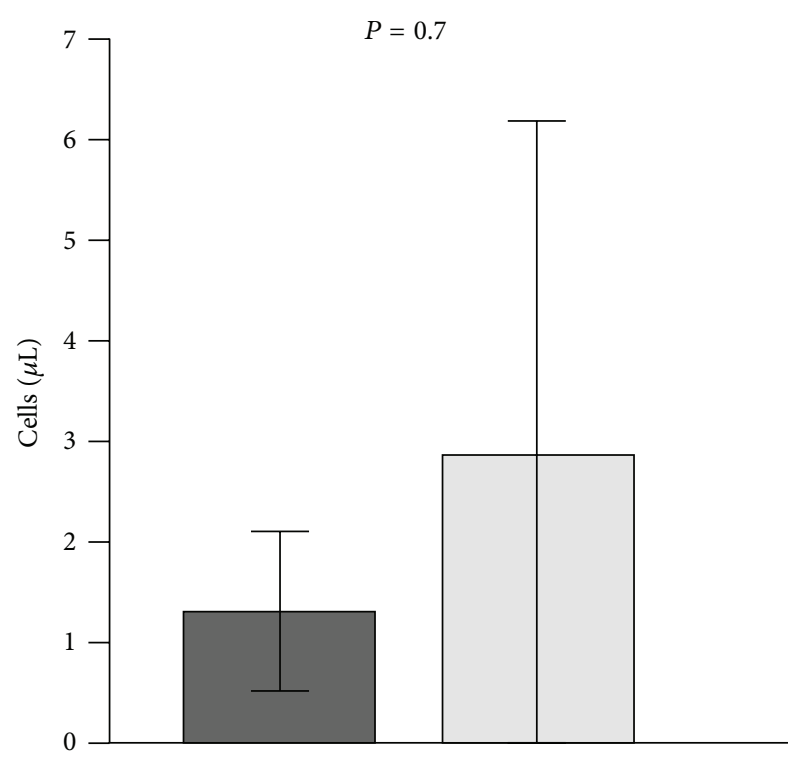

(b)

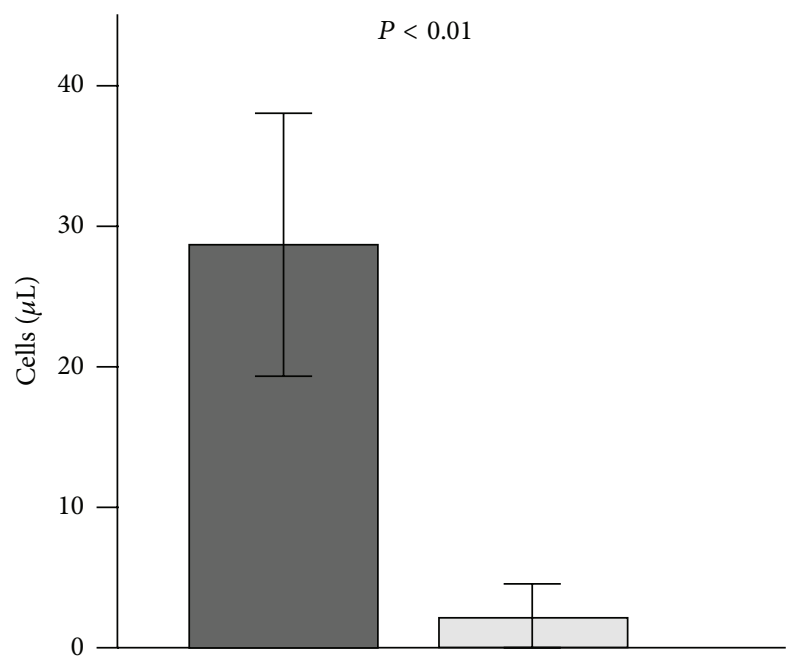

(d)

FIgURE 2: Expression of IgM, IgD, CD23, and CD27 surface molecules identifying 4 consecutive steps of B cell mauration in patients with CVID. Number of: (a) IgM+ IgD+ CD23+ CD27- (Naïve B cells), (b) IgM+ IgD- CD23- CD27-, (c) IgM- IgD- CD23- CD27-, and (d) IgM- IgD- CD23- CD27+ (switched memory B cells) in healthy controls (dark grey bar) and in CVID patients (light grey bar). Number of cells are reported on vertical axis.

airways, gastrointestinal and urinary tract infections but not with autoimmune diseases, splenomegaly, lymphadenopathy and bronchiectasis. Moreover, we observed that a low number of marginal zone B cells correlate with low levels of IgM $(12,95 \pm 17$ cells $/ \mu \mathrm{L}$ and $0,33 \pm 0,70 \mathrm{~g} / \mathrm{L}$, resp., $P=0.008)$.

\section{Discussion}

In this study, we have analyzed subsets of B cells that correspond to different phases of $\mathrm{B}$ cell maturation, in patients with CVID and in normal subjects in order to evaluate both the alterations in the number of $B$ cell subsets in this patients population and the possible correlation of these cell subsets with particular clinical features of the disease. A decrease of switched memory B cells defined as IgD- CD27+ cells and other immunophenotypic alterations have already been reported in the different classifications proposed so far [1618].

Here, we have considered the CD23 molecule [14, 21-23] as a possible marker that could add novel information on the defect of B cell maturation typical of CVID. CD23 is the low affinity receptor for IgE, a ligand of CD21, and a marker of cell maturation [21]. Its expression on B cells gradually decreases and eventually disappears after the encounter with antigens and the activation of B cells. CD23 is mainly expressed by CD27- naïve B cells, whereas its expression is reduced after 


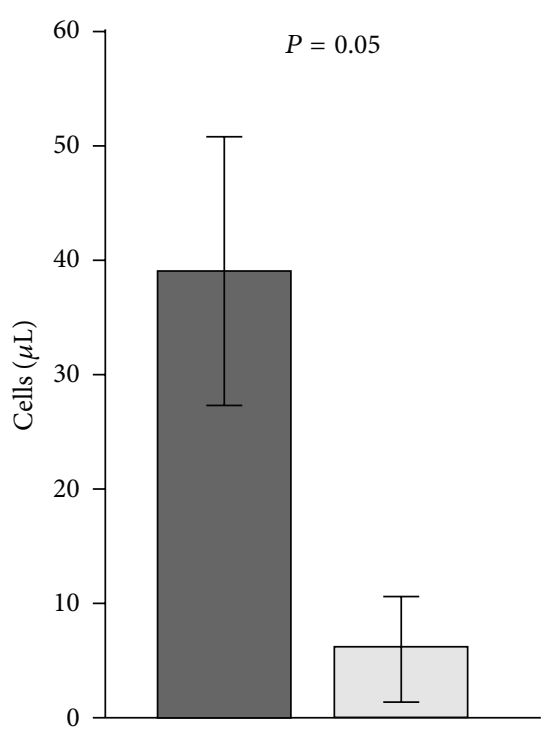

Figure 3: Switched memory B cells (according to Freiburg classification) in CVID patients. Number of IgD- CD27+ B lymphocytes (reported on vertical axis) in healthy controls (dark grey bar) and in CVID patients (light grey bar).

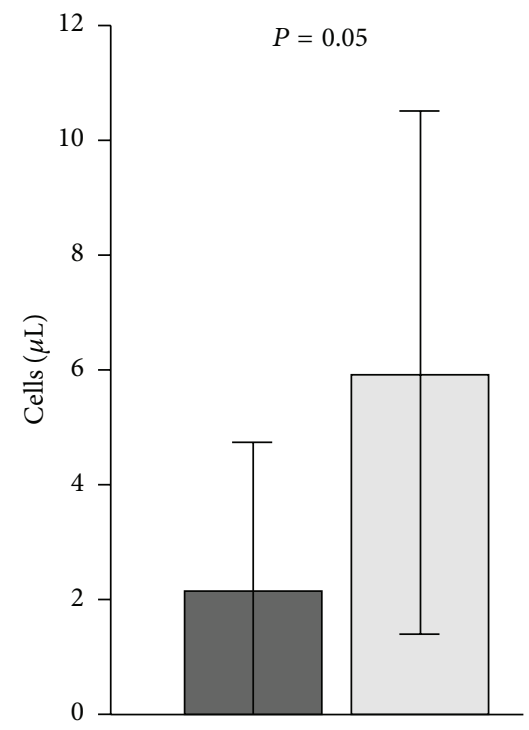

FIgURE 4: Switched memory B cells in CVID patients. Comparison between IgM- IgD- CD23- CD27+ (dark grey bar) and IgDCD27+ (light grey bar) B lymphocytes in CVID patients. Number of cells are reported on vertical axis.

the isotypic "switch." In this way, CD23 can define more clearly the maturation stages from naïve B cell to switched memory B cell. In contrast, CD27 is the marker of activated $B$ cells that have already performed somatic hypermutation $[14,15]$.

CD27 and CD23 can be considered mutually exclusive on activated $\mathrm{B}$ cells $[12,13,21]$; therefore, they can be used to identify three B lymphocyte subsets: CD23+ CD27-; CD23CD27-; and CD23-CD27+.
Although there is no significant difference between the number of CD23- CD27- B cells in patients and controls, we observed that reduced CD23-CD27- B cells were related to a history of bronchiectasis and solid tumors, in the patients' group. Moreover, in CVID patients, the marked reduction in CD23- CD27+ B cells is associated with a history of bronchiectasis and autoimmune diseases. Therefore, the low level of these two cell populations (CD23- CD27- and CD23- CD27+ B cells) is frequently associated with the presence of particular complications in CVID. On the basis of such data, we can suggest that the study of these B cell populations at diagnosis may be useful for the followup of patients in order to carefully evaluate the possible development of particular clinical features.

To better characterize the B lymphocyte subsets, we considered surface expression of IgM and IgD as markers of $B$ cell differentiation. They correlate with the level of somatic hypermutation and isotypic "switching" of B lymphocytes.

We, therefore, evaluated the $\mathrm{B}$ cell populations IgM+ IgD + CD23+ CD27- (naïve B cells) and IgM- IgD- CD23$\mathrm{CD} 27+$ (switched memory B cells); moreover, we wanted to analyse also the $B$ cell subsets that represent steps from naïve to switched memory $\mathrm{B}$ cells phenotypically characterized by the markers IgM+ IgD- CD23- CD27- and IgM- IgDCD23- CD27-.

When we consider the IgD- CD27+ B cells included in the Freiburg classification, we found a reduction of this $B$ cell subset in our cohort of patients; however, such reduction did not correlate with splenomegaly, in contrast with the report of Warnatz et al. in the group Ia of the cohort of Freiburg [16]. Moreover, we did not find that the reduction of this cell subset is associated with respiratory tract infections or other clinical features of disease.

On the contrary, IgM- IgD- CD23-CD27+ B cells were even lower than IgD- CD27+ B cells, and their number better correlated with the history of lymphadenopathy and respiratory tract infections than the number of IgD-CD27+ $B$ cells, in our cohort of patients.

Therefore, we suggest that IgM- IgD- CD23- CD27+ switched memory B lymphocytes show a better correlation with clinical features than the IgD- CD27+ B cells, confirming that the use of $\mathrm{CD} 23$ may be relevant in defining some clinical and prognostic features. From the clinical point of view, the study of this population can be useful to confirm a suspected diagnosis of CVID, in particular in the case of patients with a normal or not significantly decreased number of IgD- CD27+ B cells.

The circulating CD27+ B lymphocytes include not only switched memory B cells, but also IgM+ IgD+ CD23- CD27+ (marginal zone B cells), which represent $15 \%$ of peripheral $\mathrm{B}$ lymphocytes. These cells are derived from the marginal zone of the spleen and tend to differentiate into antibody-secreting cells in response to T-independent antigens [24, 25].

In our cohort of patients, we observed that the decrease in marginal zone B cells correlates with the increased risk to develop recurrent infections. Our preliminary data show that decreased serum levels of IgM are related to the decrease of this B cell population. However, the low level of IgM may be 


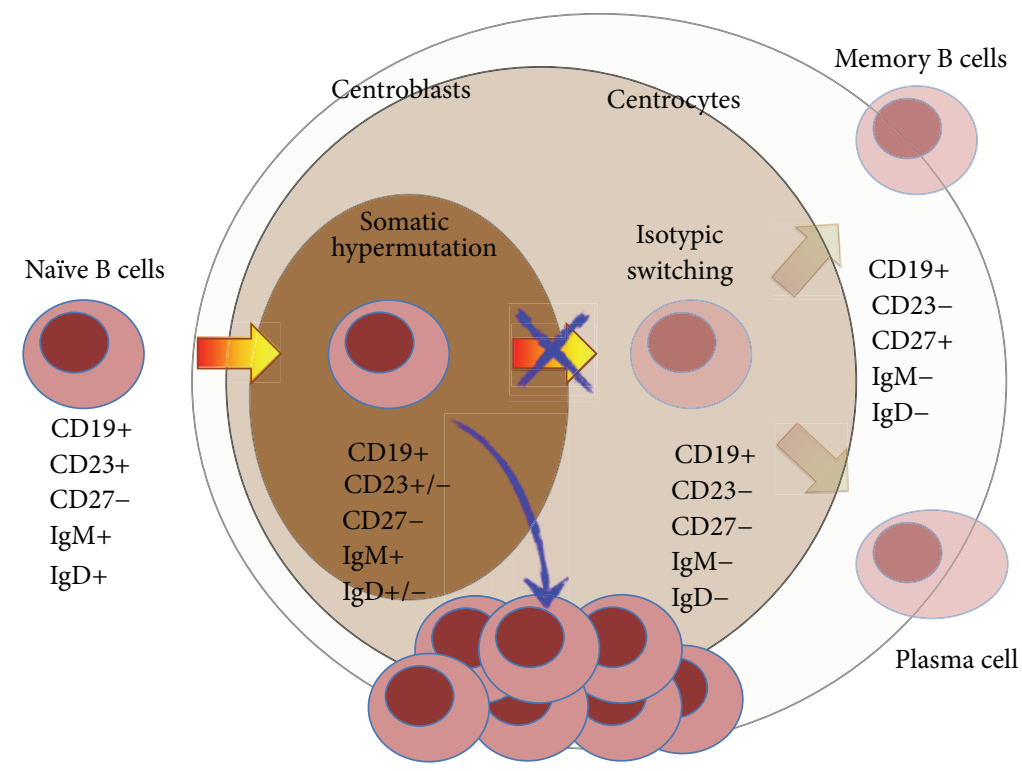

FIGURE 5: Possible explanation of B cell maturation defect in patients with CVID. The number of naïve B cells is similar in patients and controls. Since the number of IgM+ IgD- CD23-CD27- B cells is higher and that of IgM- IgD- CD23- CD27- is lower in patients compared to controls, we can hypothesize that the defect in $\mathrm{B}$ cell maturation lies at this step of $\mathrm{B}$ cell maturation.

related not only to the decrease of the marginal zone B cells, but also to the inability of such cells to produce IgM.

Finally, as shown in Figure 5, the evaluation of these different B cell populations may suggest more exactly where the $\mathrm{B}$ cell maturation defect lies.

\section{Conclusions}

Our data show that CD23-CD27- and CD23-CD27+ B cells are associated with the development of bronchiectasis, solid tumors, and autoimmune diseases, frequent complications of CVID. We suggest that the study of these cell populations could be useful for the followup of the patients in order to carefully evaluate the possible development of these clinical features.

IgD- CD27+ B cells are reduced in CVID patients compared to healthy controls without significant correlation whit the major clinical features of the disease. On the contrary IgM- IgD-CD23- CD27+ B cells are lower than IgDCD27+ B cells in patients with CVID and are associated with lymphadenopathy and airways infections.

We also observed that the decrease of marginal IgM+ IgD + CD23-CD27+ B cells is related to low level of IgM and with increased probability to develop infections.

In conclusion, we propose the introduction of CD23 in the immunophenotypical analysis of $\mathrm{B}$ cells in patients with CVID since this molecule may help in better defining the diagnosis and the characteristics of the patients.

\section{Conflict of Interests}

The authors declare that they have no conflict of interests.

\section{Authors' Contribution}

G. Patuzzo and F. Mazzi contributed equally to the work.

\section{References}

[1] M. A. Park, J. T. Li, J. B. Hagan, D. E. Maddox, and R. S. Abraham, "Common variable immunodeficiency: a new look at an old disease," The Lancet, vol. 372, no. 9637, pp. 489-502, 2008.

[2] J. H. Park, E. S. Resnick, and C. Cunningham-Rundles, "Perspectives on common variable immune deficiency," Annals of the New York Academy of Sciences, vol. 1246, no. 1, pp. 41-49, 2011.

[3] P. F. K. Yong, J. E. D. Thaventhiran, and B. Grimbacher, "A rose is a rose is a rose, but CVID is Not CVID common variable immune deficiency (CVID), what do we know in 2011?" Advances in Immunology, vol. 111, pp. 47-107, 2011.

[4] O. Kopecký and Š. Lukešová, "Genetic defects in common variable immunodeficiency," International Journal of Immunogenetics, vol. 34, no. 4, pp. 225-229, 2007.

[5] S. Goldacker and K. Warnatz, "Tackling the heterogeneity of CVID," Current Opinion in Allergy and Clinical Immunology, vol. 5, no. 6, pp. 504-509, 2005.

[6] C. Bacchelli, S. Buckridge, A. J. Thrasher, and H. B. Gaspar, "Translational Mini-Review Series on Immunodeficiency: molecular defects in common variable immunodeficiency," Clinical and Experimental Immunology, vol. 149, no. 3, pp. 401409, 2007.

[7] J. Pons, J. M. Ferrer, N. Martínez-Pomar, J. Iglesias-Alzueta, and N. Matamoros, "Costimulatory molecules and cytokine production by $\mathrm{T}$ lymphocytes in common variable immunodeficiency disease," Scandinavian Journal of Immunology, vol. 63, no. 5, pp. 383-389, 2006. 
[8] J. S. Jaffe, E. Eisenstein, M. C. Sneller et al., "T-cell abnormalities in common variable immunodeficiency," Pediatric Research, vol. 33, no. 1, supplement, pp. S24-S28, 1993.

[9] A. Giovannetti, M. Pierdominici, F. Mazzetta et al., "Unravelling the complexity of $\mathrm{T}$ cell abnormalities in common variable immunodeficiency," Journal of Immunology, vol. 178, no. 6, pp. 3932-3943, 2007.

[10] A. Giovannetti, M. Pierdominici, and F. Aiuti, "T-cell homeostasis: the dark(ened) side of common variable immunodeficiency," Blood, vol. 112, no. 2, p. 446, 2008.

[11] S. F. Gonzalez, S. E. Degn, and L. A. Pitcher, "Trafficking of B cell antigen in lymph nodes," Annual Review of Immunology, vol. 29, pp. 215-233, 2011.

[12] M. Shapiro-Shelef and K. C. Calame, "Regulation of plasmacell development," Nature Reviews Immunology, vol. 5, no. 3, pp. 230-242, 2005.

[13] J. Y. Bonnefoy, S. Lecoanet-Henchoz, J. P. Aubry, J. F. Gauchat, and P. Graber, "CD23 and B-cell activation," Current Opinion in Immunology, vol. 7, no. 3, pp. 355-359, 1995.

[14] U. Klein, R. Küppers, and K. Rajewsky, "Evidence for a large compartment of IgM-expressing memory B cells in humans," Blood, vol. 89, no. 4, pp. 1288-1298, 1997.

[15] S. G. Tangye and K. L. Good, "Human IgM+CD27+B cells: memory B cells or “memory" B cells?” Journal of Immunology, vol. 179, no. 1, pp. 13-19, 2007.

[16] K. Warnatz, A. Denz, R. Dräger et al., "Severe deficiency of switched memory B cells (CD27+IgM-IgD-) in subgroups of patients with common variable immunodeficiency: a new approach to classify a heterogeneous disease," Blood, vol. 99, no. 5, pp. 1544-1551, 2002.

[17] B. Piqueras, C. Lavenu-Bombled, L. Galicier et al., "Common variable immunodeficiency patient classification based on impaired B cell memory differentiation correlates with clinical aspects," Journal of Clinical Immunology, vol. 23, no. 5, pp. 385400, 2003.

[18] C. Wehr, T. Kivioja, C. Schmitt et al., "The EUROclass trial: defining subgroups in common variable immunodeficiency," Blood, vol. 111, no. 1, pp. 77-85, 2008.

[19] N. Taubenheim, M. Von Hornung, A. Durandy et al., "Defined blocks in terminal plasma cell differentiation of common variable immunodeficiency patients," Journal of Immunology, vol. 175, pp. 5498-5503, 2005.

[20] M. E. Conley, L. D. Notarangelo, and A. Etzioni, "Diagnostic criteria for primary immunodeficiencies. Representing PAGID (Pan-American Group for Immunodeficiency) and ESID (European Society for Immunodeficiencies)," Clinical Immunology, vol. 93, no. 3, pp. 190-197, 1999.

[21] D. Veneri, R. Ortolani, M. Franchini, G. Tridente, G. Pizzolo, and A. Vella, "Expression of CD27 and CD23 on peripheral blood B lymphocytes in humans of different ages," Blood Transfusion, vol. 7, no. 1, pp. 29-34, 2009.

[22] R. H. J. Verstegen, M. A. A. Kusters, E. F. A. Gemen, and E. De Vries, "Down syndrome B-lymphocyte subpopulations, intrinsic defect or decreased T-lymphocyte help," Pediatric Research, vol. 67, no. 5, pp. 563-569, 2010.

[23] H. J. Gould, R. Reljic, and J. Shi, IgE Homeostasis: Is CD23 the Safety Switch? IgE Regulation: Molecular Mechanisms, John Wiley \& Sons, New York, NY, USA, 1997.

[24] M. Seifert and R. Küppers, "Molecular footprints of a germinal center derivation of human $\operatorname{IgM}+(\operatorname{IgD}+) \mathrm{CD} 27+\mathrm{B}$ cells and the dynamics of memory B cell generation," Journal of Experimental Medicine, vol. 206, no. 12, pp. 2659-2669, 2009.
[25] F. Martin and J. F. Kearney, "Marginal-zone B cells," Nature Reviews Immunology, vol. 2, pp. 323-335, 2002. 


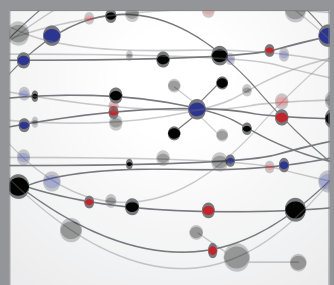

The Scientific World Journal
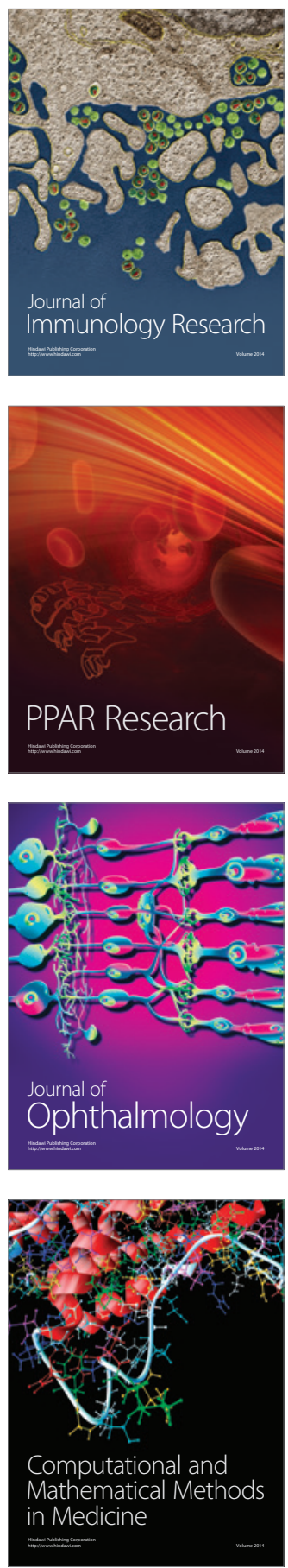

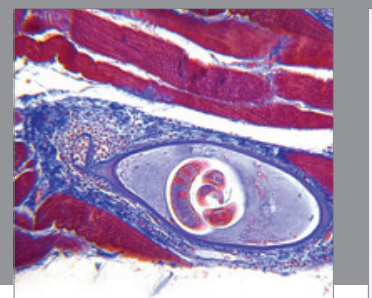

Gastroenterology

Research and Practice
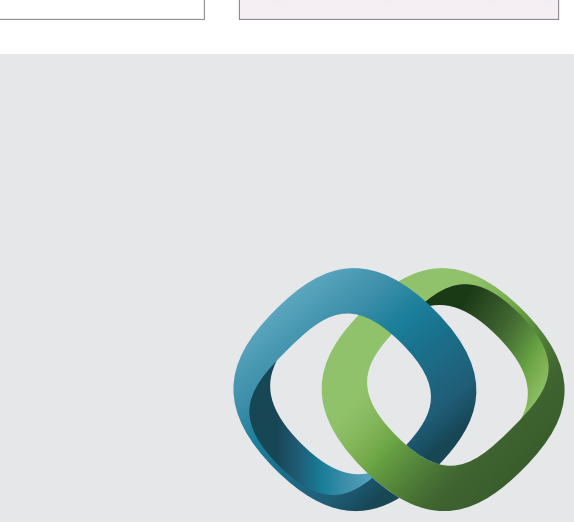

\section{Hindawi}

Submit your manuscripts at

http://www.hindawi.com
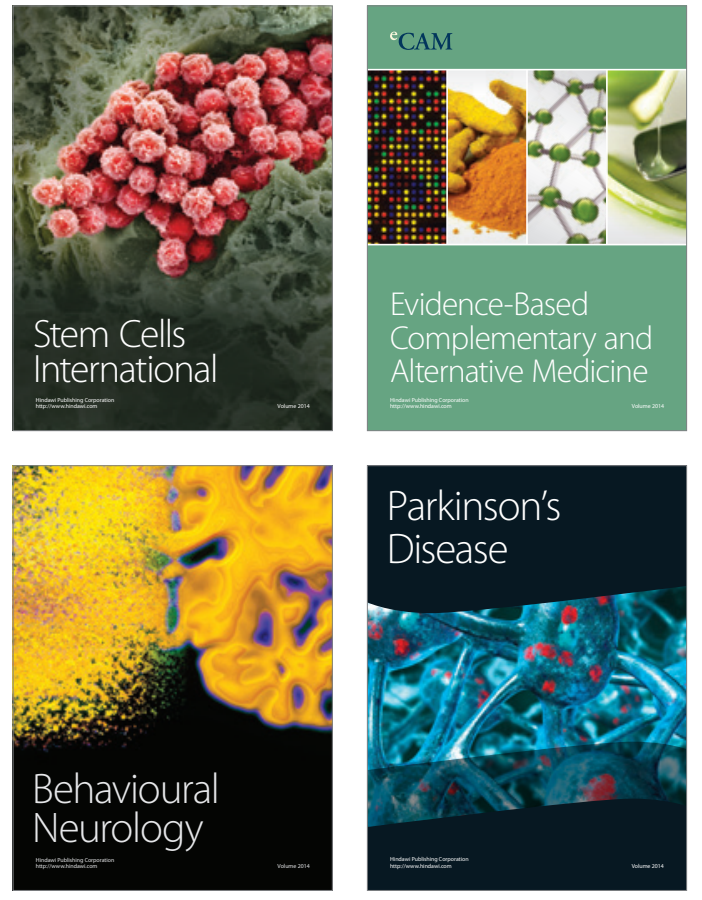
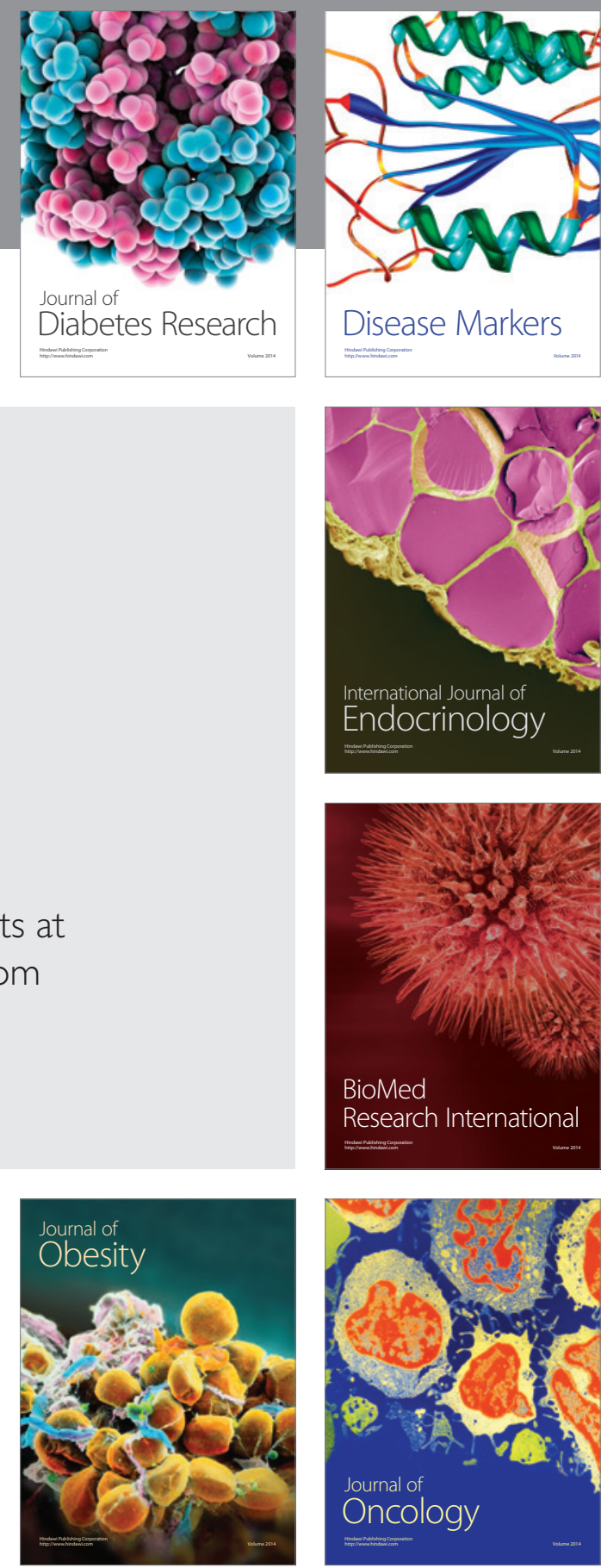

Disease Markers
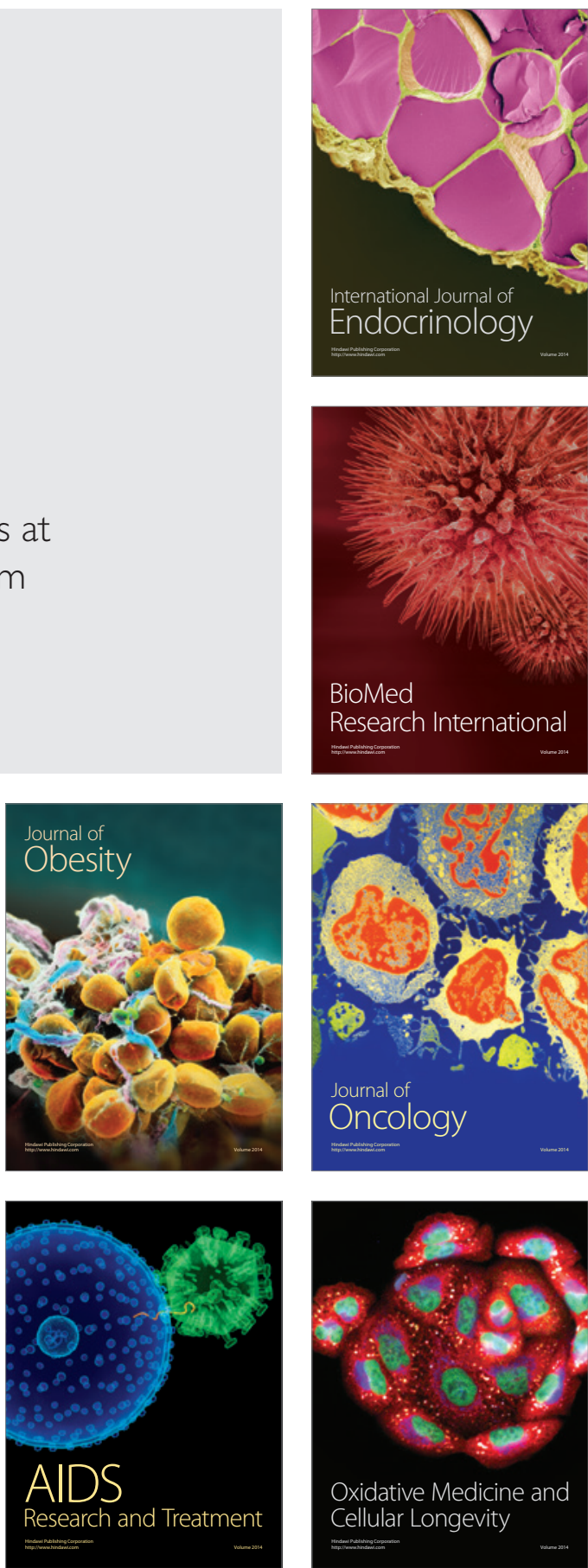